Received: 2019.06.09 Accepted: 2019.09.06 Published: 2019.12.03

Authors' Contribution: Study Design A Data Collection B Statistical Analysis C Data Interpretation D Manuscript Preparation E Literature Search F Funds Collection $G$

Corresponding Author: Conflict of interest:

\title{
Anatomy of a Suicide: A Case Report
}

\author{
EF 1 Sebastian D. Sgardello \\ AG 2 Michel Christodoulou \\ BEF 1 Ziad Abbassi
}

1 Department of General Surgery, Hospital Center Valais Romand - Hospital Sion, Sion, Switzerland

2 Department of Thoracic Surgery, Hospital Center Valais Romand - Hospital Sion, Sion, Switzerland

\begin{abstract}
Patient: Female, 52-year-old
Final Diagnosis: Left pneumothorax

Symptoms: Dyspnea

Medication: -

Clinical Procedure: -

Specialty: Surgery

Objective: Unusual clinical course

Background: Penetrating neck injuries (PNI) have a relatively low incidence constituting just $1.6 \%$ to $3.0 \%$ of overall suicide attempts. Nonetheless, the anatomical challenges as well as the likelihood of vascular and airway lesions make it one of the most lethal injury types of all Abbreviated Injury Scale regions.

Traditional PNI management which divides PNI into anatomical zones is being reconsidered in light of high numbers of negative surgical explorations, weak correlation between the area of wounds and organ injury and significantly longer hospitalizations.

Case Report: A 52-year-old female was admitted after a self-inflicted, right para tracheal stab wound. A cervico-thoracic computed tomography (CT) scan excluded vascular and other organ lesions. A right pneumothorax was treated with a chest drain and a right exploratory cervicotomy was performed. A pharyngoscopy and an esophagoscopy showed no lesions.

Conclusions: $\quad$ Advanced Trauma Life Support (ATLS) principles determine the initial assessment of PNI. Invasive airway management was required if orotracheal intubation is unfeasible. Hemodynamically unstable patients with platysma, vascular or aerodigestive lesions require surgery. Laryngotracheal injuries require panendoscopy and bronchoscopy prior to surgical exploration. Pharyngo-esophageal injuries may be treated conservatively. Esophageal lesions require timing dependent surgery. Recently, a "no zone" approach irrespective of anatomical classification shows improved results in stable PNI. Multidetector helical CT with angiography (MDCT-A) significantly reduces negative exploratory surgery. Consensus regarding the best management of PNI is shifting, as increasing evidence suggests a "no-zone" approach is more beneficial and cost effective.
\end{abstract}

MeSH Keywords: Neck Injuries • Pneumothorax • Schizotypal Personality Disorder • Suicide, Attempted

Full-text PDF: https://www.amjcaserep.com/abstract/index/idArt/917993

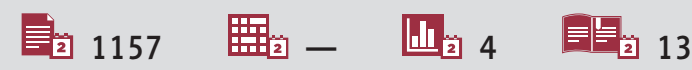




\section{Background}

Penetrating neck injuries (PNI) are defined as neck injuries that penetrate the platysma and make up $5 \%$ to $10 \%$ of traumatic injuries in adults [1]. Furthermore, in comparison to attempted suicides, PNI constitute $1.6 \%$ to $3.0 \%$ of overall suicide attempts.

Nonetheless, the anatomical challenges as well as the likelihood of vascular and airway lesions make it one of the most lethal injury types of all Abbreviated Injury Scale regions. [2]

Over the last decade, mortality in both civilian and military populations have been approximately $5 \%$ with death by exsanguination being the most common cause of immediate death. Mortality from penetrating laryngotracheal trauma is $20 \%$ whilst mortality from pharyngoesophageal injuries reaches $22 \%[1,3]$

Potential lesions involve the blood vessels at the root of the neck, mediastinum, internal mammary arteries, vena azygos as well as intercostal vessels which may result in hematomas of the mediastinum, of the thoracic apex as well as hemo-pneumothorax.

Management of PNI has evolved considerably, moving from a widely accepted, mandatory exploratory surgical approach to a more selective, multidisciplinary approach due to high negative exploration rates.

This has led to the creation of a zone-based approach to stable PNI thus allowing the multidisciplinary team to classify PNI into anatomic zones.

More recently however, and as a result of improvement in diagnostic imaging, a "no zone" strategy has been developed. Patients are classified unstable or stable irrespective of the zone of neck injury. Unstable patients are treated in the operating room whilst stable but symptomatic patients undergo multidetector computed tomography angiography (MDCT-A) which in combination with a clinical examination, indicate the need for further diagnostic tests or if surgical intervention is needed.

A rare case of a suicide attempt by stabbing in the neck resulting in a contralateral pneumothorax is presented [4].

\section{Case Report}

A 52-year-old female consulted the Emergency Department of our institution because of a self-inflicted, right para tracheal stab wound to the neck with a $10 \mathrm{~cm}$ kitchen knife (Figures 1, 2). The patient suffers from a schizotypal personality disorder associated with depression and has a history of

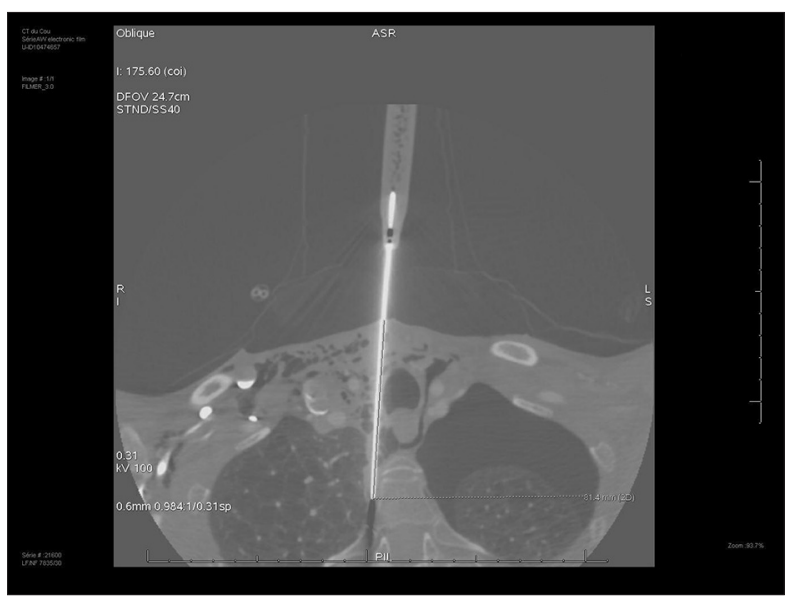

Figure 1. Intravenous contrast-enhanced computed tomography (CT) of the thorax and cervical region. Axial CT image showing the trajectory of the blade through the cervical and mediastinal region without lesions to vessels or vital organs.

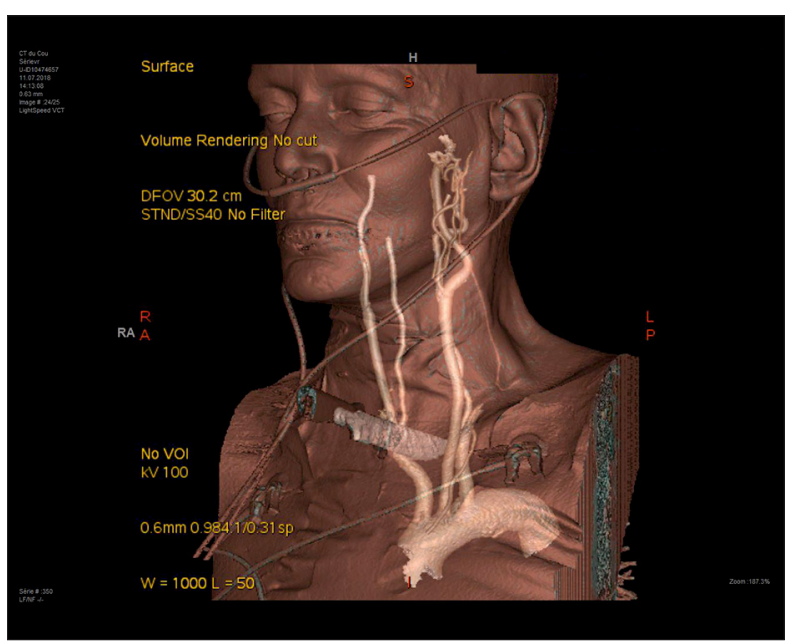

Figure 2. Three-dimensional volume rendition shows the oblique trajectory of the blade, between the brachiocephalic trunk and the left common carotid.

2 suicide attempts, both violent and non-violent via phlebotomy and prescription drug overdose respectively.

Upon arrival the patient was hemodynamically stable, with no active bleeding. Vital parameters where normal and a physical examination showed a left pneumothorax. A cervico-thoracic CT scan excluded any vascular lesions as well as any lesions to internal organs (Figures 3, 4). A naso-fiberscope showed no vocal cord paralysis. Blood tests where unremarkable. Hemoglobin levels were stable around $100 \mathrm{~g} / \mathrm{L}$.

In the operating room, a $28 \mathrm{CH}$ chest drain was inserted and a right exploratory cervicotomy was performed resulting in an iatrogenic right pneumothorax which was drained with a $28 \mathrm{CH}$ 


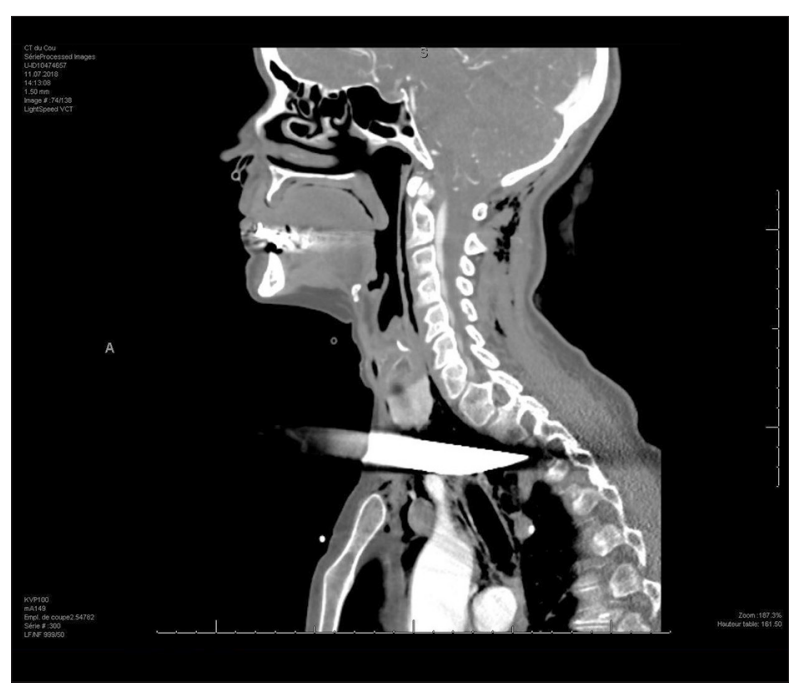

Figure 3. A sagittal computed tomography (CT) scan showing the depth of penetration of the knife blade.

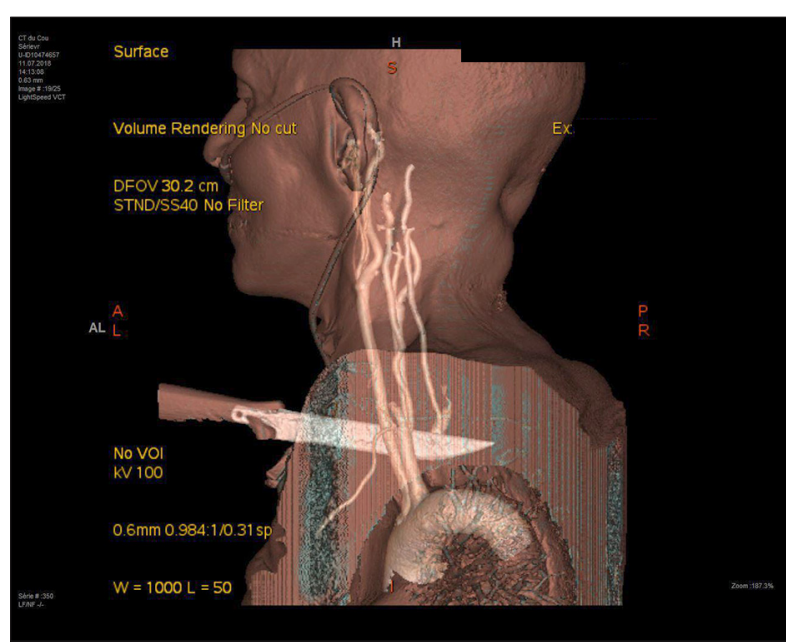

Figure 4. A 3-dimensional volume rendition showing the depth of penetration of the knife blade.

chest drain. The knife was removed and diffuse bleeding from the thyroid gland was stopped. A pharyngoscopy and an esophagoscopy showed no other lesions. The patient was then admitted to the subintensive care unit where the right and left chest tubes were removed at post-operative day 1 and day 2 respectively. A wide spectrum prophylactic antibiotic treatment of co-amoxicillin $2.2 \mathrm{~g}$ intravenous (IV) was continued for a total of 7 days and at post-operative day 3, following a psychiatric evaluation, the patient was transferred to the Psychiatric Department of our hospital.

\section{Discussion}

Historically, the management of PNI has followed a 3-zone division. Zone 1 is between the sternal notch and the cricoid cartilage, zone
2 is between the cricoid cartilage and the angle of the mandible and zone 3 is from the angle of the mandible to the skull base.

However, this approach is being reconsidered in light of the high numbers of negative surgical explorations in zone 2, hemodynamically stable injuries (13\% to $19 \%$ ) as well as a weak correlation between the area of wounds and actual organ injury and significantly longer hospitalizations due to expensive, labor, and resource intensive procedures. Additionally, multiple or trans cervical injuries might be impossible to correctly classify into specific zones [2,5].

Our patient epitomized this, presenting a right paramedian point of entry with an anteroinferior trajectory, lateral to the trachea, inferior to the thyroid gland, medial to the carotid artery, and above the celiac plexus. The point of the blade was adjacent to the D3 vertebrae.

Advanced Trauma Life Support (ATLS) protocols guide the primary assessment of PNI. An intact platysma defines the wound as being superficial. Otherwise surgical consultation is required, ideally with the otolaryngology team. Cervical spine collars are not recommended except in the presence of focal neurology or a strong clinical suspicion of spinal injury. Airway management initially includes clinical examination for signs of aero digestive tract injury. Invasive airway management should be performed in cases where orotracheal intubation is impossible [6].

The decision to operate is based on the patient's physiological status and clinical findings. Hemodynamically unstable patients with violation of the platysma and lesions to vascular or aero digestive structures (hard signs), are in need of surgical intervention $[7,8]$

Half of all deaths from PNI are caused by exsanguination [6]. Foley balloon catheter tamponade might be used in hemorrhage not amenable by simple compression. Vascular injuries require an assessment with a vascular surgeon and zone 1 injuries could require a cardiothoracic approach. Lesions to the internal carotid artery have a superior outcome if repaired as opposed to artery ligation, irrespective of preoperative focal neurological deficits was present $[10,9]$

Laryngotracheal injuries require panendoscopy and bronchoscopy prior to surgical exploration. Conservative treatment may be indicated in cases of small mucosal defects or non-displaced fractures of the larynx. Open repair is needed in the presence of skeletal fractures or injuries to soft tissues $[8,6,11]$.

Intravenous antibiotics and surgical nutrition are needed in patients with pharyngo-esophageal injuries. Esophageal injury or perforation require operative repair which is timing dependent $[7,8]$. 
If treatment is performed within 12 hours of injury, suture repair and drainage may be performed. After 12 hours of injury, a planned delayed repair should be performed because of the increase in morbidity and mortality associated with debridement and drainage $[8,12]$.

More recently, a "no zone" approach irrespective of anatomical classification has shown improved outcomes in stable PNI. PNI can be managed based on an individual basis according to clinical findings and the patient's physiological status $[6,9,11]$.

Furthermore, multidetector helical computed tomography with angiography (MDCT-A) significantly reduces negative exploratory surgery $[7,8]$.

However, some studies report a sensitivity in diagnosing pharyngo-esophageal injuries as low as $53 \%$ meaning that additional imaging is often required [12].

\section{References:}

1. Shiroff A, Gale S, Martin N et al: Penetrating neck trauma: A review of management strategies and discussion of the "no zone" approach. Am Surg, 2013; 79(1): 23-29

2. Triggiani E, Belsey R: Oesophageal trauma: Incidence, diagnosis, and management. Thorax, 1977 ;32: 241-49

3. Asensio JA, Berne J, Demetriades D et al: Penetrating esophageal injuries: Time interval of safety for preoperative evaluation - how long is safe? J Trauma, 1997; 43: 319-24

4. Sgardello SD, Christodoulou M, Abbassi Z: Anatomy of a suicide. Eur Surg Res, 2019; 60(Suppl. 2): 1-104. Abstract.

5. Feliciano DV: Management of penetrating injuries to carotid artery. World J Surg, 2001; 25: 1028-35

6. Osborn TM, Bell RB, Qaisi W, Long WB: Computed tomographic angiography as an aid to clinical decision making in the selective management of penetrating injuries to the neck: A reduction in the need for operative exploration. J Trauma, 2008; 64: 1466-71
The MDCT-A results may warrant further investigations with angiography, bronchoscopy, contrast swallow/flexible esophagoscopy or surgical exploration [13].

Nonetheless, the management of PNI requires a multidisciplinary approach. Studies support a selective operative management of stable patients with a no-zone approach stratified according to patient stability. No clear indication exists for mandatory surgical exploration, especially with zone II injuries $[1,3,13]$.

\section{Conclusions}

At this time, consensus regarding the best management of PNI is shifting as increasing evidence suggests a "no-zone" approach is more beneficial and cost effective, namely in reducing negative exploratory surgery.

7. Feliciano DV: Penetrating cervical trauma. Current concepts in penetrating trauma, IATSIC Symposium, International Surgical Society, Helsinki, Finland, August 25-29, 2013. World J Surg, 2015; 39: 1363-72

8. Burgess CA, Dale OT, Almeyda R, Corbridge RJ: An evidence-based review of the assessment and management of penetrating neck trauma. Clin Otolaryngol, 2012; 37: 44-52

9. Madiba TE, Muckart DJ: Penetrating injuries to the cervical oesophagus: Is routine exploration mandatory? Ann R Coll Surg Engl, 2003; 85: 162-66

10. Mandavia DP, Qualls S, Rokos I: Emergency airway management in penetrating neck injury. Ann Emerg Med, 2000; 35: 221-25

11. Fogelman MJ, Stewart RD: Penetrating wounds of the neck. Am J Surg, 1956; 91: 581-99

12. Hussain Zaidi SM, Ahmad R: Penetrating neck trauma: A case for conservative approach. Am J Otolaryngol, 2011; 32: 591-96

13. Low GM, Inaba K, Chouliaras K et al: The use of the anatomic 'zones' of the neck in the assessment of penetrating neck injury. Am Surg, 2014; 80: 970-74 